\section{Diagnosing concussion}

In a $C M A J$ practice article, Tator states that "the diagnosis of concussion is not easy." However, the current diagnostic criteria for concussion ${ }^{2}$ are remarkably rudimentary and state that a single symptom (from a 22-item Likert scale) experienced after head shake, should prompt a clinician to diagnose concussion and institute treatment. Tator does not discuss the difficulty caused by the lack of specificity of the diagnostic criteria for concussion, as they are currently defined. ${ }^{2}$ Individuals with whiplash, depression, benign vertigo and other disorders all meet the diagnostic criteria for concussion. The problem is that once feelings of everyday life are attributed to a brain injury, concussion is not easily "undiagnosed."

Tator describes removal from play, work and school as the most important aspects of initial management, but does not provide a citation. Current literature suggests that rest is not effective for the management of concussion. ${ }^{3}$ Tator also states that all symptoms have to have completely disappeared before an individual is allowed to return to play, work or school. Symptoms of concussion are present in healthy people - the average symptom score for healthy, uninjured people on the concussion Likert scale is not zero. ${ }^{4}$ The treatment goal of being asymptomatic is not evidencedbased, and has the potential for locking people in an isolated existence, while they wait for symptoms that may have nothing to do with brain injury to disappear. Tator has not discussed the substantial influence that psychological factors have in the genesis and perpetuation of postconcussion syndrome.

Of great concern are the consequences of concussion listed in Tator's article. ${ }^{1}$ Second-impact syndrome is a dubious condition which may or may not even exist. ${ }^{5}$ The paper cited in Tator's article states "the scientific evidence to support this concept is nonexistent, and belief in the syndrome is based upon the interpretation of anecdotal cases more often than not, lacking sufficient clinical detail to make definitive statements. The fear of this condition has driven many of the current return-toplay guidelines following concussion." 6

Articles such as Tator's raise fear regarding a condition with an excellent prognosis. A balanced statement that indicates that up to $90 \%$ of concussed individuals get better without any intervention within 7 to 10 days, ${ }^{7}$ would help readers understand that concussion is a benign condition, more analogous to the common cold than some sinister dementing condition.

\section{Neil Craton BSc MHK MD Dip Sport Med, Oliver Leslie MD}

Sport medicine physician, Legacy Sport Medicine (Craton), Winnipeg, Man.; Department of Family Medicine (Craton); Prograduate Medical Education (Leslie), University of Manitoba, Winnipeg, Man.

\section{References}

1. Tator $\mathrm{CH}$. Concussions and their consequences: current diagnosis, management and prevention. CMAJ 2013; 185:975-9.

2. McCrory P, Meeuwisse WH, Aubry M, et al. Consensus statement on concussion in sport: the 4th International Conference on Concussion in Sport held in Zurich, November 2012. Br J Sports Med 2013;47:250-8

3. de Kruijk JR, Leffers P, Meerhoff S, et al. Effectiveness of bed rest after mild traumatic brain injury: a randomised trial of no versus six days of bed rest. J Neurol Neurosurg Psychiatry 2002;73:167-72

4. Alla S, Sullivan SJ, McCrory P. Defining asymptomatic status following sports concussion: fact or fallacy? Br J Sports Med 2012;46:562-9.

5. McCrory P. Does second impact syndrome exist? Clin J Sport Med 2001;11:144-9.

6. McCrory P, Davis G, Makdissi M. Second impact syndrome or cerebral swelling after sporting head injury. Curr Sports Med Rep 2012;11:21-3.

7. McCrory P, Johnston K, Meeuwisse W, et al. Summary and agreement statement of the 2nd International Conference on Concussion in Sport, Prague 2004. Br J Sports Med 2005;39:196-204.

CMAJ 2013. DOI:10.1503/cmaj.113-2149

Tator's ${ }^{1}$ review of concussion is welcomed. When writing about concussions, the popular press has created a belief held by the public and many medical professionals that a cause-and-effect link has been shown between repeated or frequent concussions or subconcussive blows and chronic traumatic encephalopathy (CTE). This very question was hotly debated in Zurich in 2012, following a presentation on CTE by Dr. McKee, from the Boston University Center for the Study of Traumatic
Encephalopathy. ${ }^{2}$ The audience contested the idea that repeated concussions and CTE were cause-and-effect, although the relationship between the 2 entities was readily admitted. The consensus statement $^{3}$ reported on CTE as follows:

"Clinicians need to be mindful of the potential for long-term problems in the management of all athletes. However, it was agreed that chronic traumatic encephalopathy (CTE) represents a distinct tauopathy with an unknown incidence in athletic populations. It was further agreed that a cause and effect relationship has not as yet been demonstrated between CTE and concussions or exposure to contact sports. At present, the interpretation of causation in the modern CTE case studies should proceed cautiously. It was also recognised that it is important to address the fears of parents/athletes from media pressure related to the possibility of CTE.'

Because there is no current in vivo marker or easily accessible method for following the markers of CTE in an athlete, the diagnosis remains a postmortem diagnosis. Currently, there are longitudinal studies proposed at various centres in North America to follow markers and radiological studies in high-risk groups to develop tools to identify and predict the possibility of CTE or chronic neurological impairment in live participants. These studies will require lengthy periods of time, monetary resources and the commitment of investigators and participants to provide a better answer to the possibility of cause-and-effect with respect to CTE and repeated mild traumatic brain injury.

As a clinician involved in the care of sport-related concussions, I deal with parents and athletes who raise the concern of CTE and chronic neurologic impairment at almost every encounter. I believe that to share with parents and athletes the Concussion in Sport Group's statement ${ }^{3}$ on CTE is more appropriate than to promulgate the belief of proven cause-and-effect.

\section{David J. Rhine MD}

Physician, Faculty of Medicine, University of British Columbia Okanagan, Kelowna, BC 